\title{
Screening for Very Mild Subcortical Vascular Dementia Patients Aged 75 and Above Using the Montreal Cognitive Assessment and Mini-Mental State Examination in a Community: The Kurihara Project
}

\author{
Mari Kasai Kenichi Meguro Kei Nakamura Masahiro Nakatsuka \\ Yoshitaka Ouchi Naofumi Tanaka \\ Department of Geriatric Behavioral Neurology, Tohoku University Graduate School of Medicine, \\ Sendai, Japan
}

\section{Key Words}

Montreal Cognitive Assessment • Clinical Dementia Rating • Mild cognitive impairment •

Subcortical vascular dementia $\cdot$ Community

\begin{abstract}
Aims: To examine the effectiveness of the Montreal Cognitive Assessment (MoCA) to screen people with mild cognitive impairment $(\mathrm{MCl})$, to associate the MoCA score with the presence of infarction, and to detect the characteristics of people with very mild subcortical vascular dementia (vmSVD). Methods: 392 out of 886 community dwellers aged 75 years and above living in Kurihara, Northern Japan, agreed to participate in our study; 164 scored a Clinical Dementia Rating (CDR) of 0 (healthy), 184 scored a CDR of 0.5 (MCI) and 44 scored a CDR of $1+$ (dementia). The participants scoring a CDR of 0.5 were divided into 2 subtypes: 37 had vmSVD and 147 had other types of dementia. The objective variables were the total MoCA, the MoCA subscale and the Mini-Mental State Examination (MMSE). Results: There was a difference in the MoCA and MMSE scores between the 3 CDR groups. The MoCA score overlapped in participants with CDR 0 and 0.5. There were significant CDR effects, while there were no significant infarction effects for the MoCA and MMSE. vmSVD participants had lower scores on the total MoCA, the MoCA attention subscale and MMSE than healthy elderly people and participants with other types of dementia. Conclusion: Our results suggested that MMSE performed rather well and that the MoCA is not superior to MMSE in MCl and vmSVD participants aged 75 and above in a community.




\section{Introduction}

Mild cognitive impairment (MCI) [1,2] or a Clinical Dementia Rating (CDR) of 0.5 [3, $4]$ is a transitional state between normal/healthy and dementia. People with MCI or CDR 0.5 show some neuropsychological deficiencies, such as loss of memory, attention, and visuospatial and executive functions [5-9]. This state should be detected for a possible prediction of dementia. CDR 0.5 with cerebrovascular disease (CVD) [10] was defined as vascular cognitive impairment no dementia (VCI-ND), and VCI-ND with neurological signs included subcortical vascular dementia (SVD) [11]. In a previous study, 37.5\% of the participants scoring a CDR of 0.5 had VCI-ND [12]. 7\% of those VCI-ND participants met the criteria for SVD. The participants with SVD were at a very mild state (CDR 0.5) and then progressed to a mild state (CDR 1) due to poor control of vascular risk factors [11]. Individuals with very mild SVD (vmSVD) are treatable, although most of them are hidden within the community [11].

Since the CDR requires information from collateral sources, it is difficult to rate people who live alone [13]. A supplementary neuropsychological screening test is needed for an early detection of individuals with CDR 0.5 in a community. The Mini-Mental State Examination (MMSE) [14] is a screening test for the detection of dementia, but it is not suitable to detect very mild cognitive impairment before dementia.

The Montreal Cognitive Assessment (MoCA) was developed by Nasreddine et al. [15] in 2005 to detect people with amnestic MCI in a clinic-based study. In community-based studies, people with amnestic MCI or Alzheimer's disease had significantly lower MoCA scores than healthy elderly [16]. Since low educational levels influence the MoCA score [15, 17-19], one point is added to the original method when a participant has a low educational level [15, 17]. However, there have been no detailed reports on large populations aged 75 years and more (75+) using the MoCA so far.

A previous study suggested that the attention subscale of the MoCA was uniquely predictive of rehabilitation success for geriatric inpatients, and attentional abilities may need to be attended to specifically in poststroke rehabilitation [20]. The MoCA attention subscale consists of a forward digit span, a backward digit span, vigilance and serial 7s [15]. The forward digit span test studies the short-term retention capacity [21], and the backward digit span test requires people to hold information in mind while performing a mental operation (i.e. working memory) [21]. The vigilance test examines the ability to sustain and focus attention, and the serial $7 \mathrm{~s}$ test is intended to measure attention and calculation [21].

Regarding CVD, Godefroy et al. [22] reported that among patients who suffered an acute poststroke, those with cognitive impairment scored significantly lower using the MoCA than patients without cognitive impairment. A recent report suggested that the MoCA is more sensitive than MMSE in patients with subcortical VCI [23]. However, so far there has been no report studying the relationship between the MoCA attention subscale and CVD.

As described above, there have been no reports on people aged 75+ with a CDR of 0.5 using the MoCA in a community. In addition, there have been no reports on participants with chronic cerebral infarction and vmSVD using neuropsychological screening tests such as the MoCA, specifically the MoCA attention subscale, in a community.

The aims of this study were (1) to examine the effectiveness of the MoCA to screen people aged $75+$ with a CDR of 0.5 (MCI) in a community, (2) to associate the MoCA score with the presence of infarction, and (3) to detect the characteristics of vmSVD participants. 
Fig. 1. Protocol for the selection of participants.

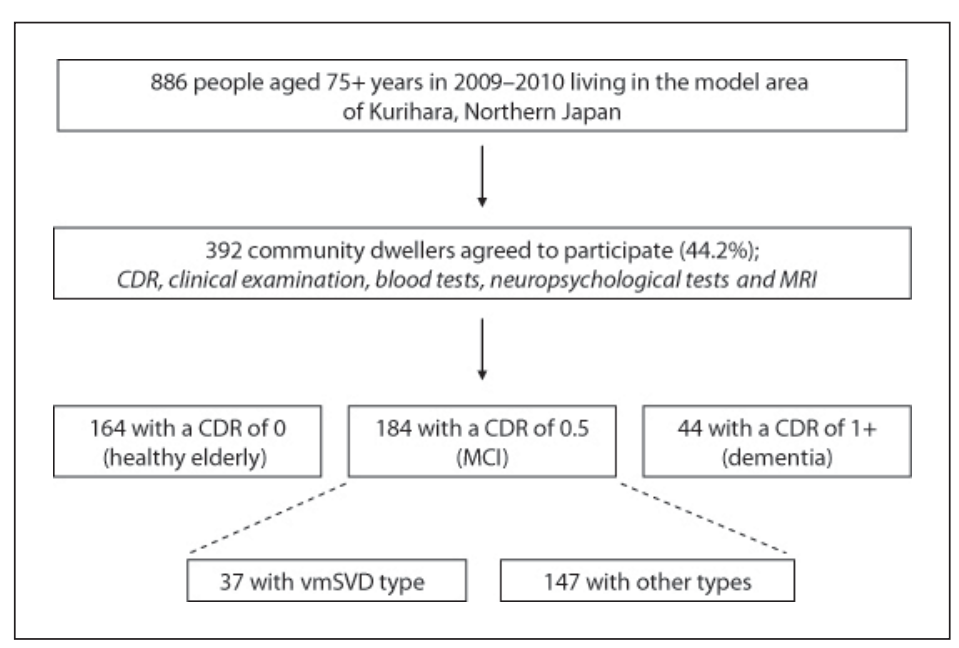

\section{Patients and Methods}

\section{Participants}

From September 2009 to December 2010, 886 people aged 75+ living in the model area of Kurihara, Northern Japan, were approached to participate in the study. Figure 1 shows the recruitment protocol for selecting the participants. 392 of the 886 community dwellers agreed to participate (44.2\%); 164 scored a CDR of 0 , which means they are healthy elderly, 184 scored a CDR of 0.5 , which means they have MCI, and 44 scored a CDR of $1+$, which means they have dementia. Participants with a CDR of $1+$ included those with a CDR of $1(n=29)$, CDR of $2(n=13)$ and CDR of $3(n=2)$. We obtained written informed consent from all participants. We utilized the CDR, clinical examination, blood tests, neuropsychological tests and magnetic resonance imaging (MRI). The participants with a CDR of 0.5 were divided into 2 subgroups: 37 of the 184 with a CDR of 0.5 had vmSVD and 147 had other types of dementia. The procedures of CDR assessment and MRI, and the diagnostic criteria for dementia and CDR 0.5 subtypes (vmSVD and other types of dementia) are described below. We excluded 1 participant (age 85 years, education 8 years) with other types of dementia from the analyses, since she did not complete the MoCA as she refused to reply to the delayed recall subscale of the MoCA.

\section{CDR Assessment}

A clinical team comprised of skilled physicians (neurologists and one psychiatrist) and skilled public health nurses determined the CDR for each participant $[3,4]$; they were blinded to the cognitive test results and they used the full questionnaire of the Japanese version of the CDR scoring sheet [24]. Before the interviews with the physicians, the public health nurses visited the participants' homes in order to evaluate their daily activities. Observations from family members regarding the participants' lives were described in a semistructured questionnaire. Participants who lived alone were visited frequently by public health nurses in order to evaluate their daily lives. The physicians interviewed the participants to assess episodic memory, orientation and judgment. Finally, with reference to the information provided by the family members, the CDR for each of the participants was determined at a joint meeting of the physicians and public health nurses. One of the authors (K.M.) is certified as a CDR rater from the Washington University School of Medicine, Alzheimer's Disease Research Center, Memory and Aging Project. We used CDR scores and CDR-sum of box (CDR-SOB) scores. 
Magnetic Resonance Imaging

We used a 1.5-Tesla MRI (Achiva 1.5T or Intera 1.5T, Philips Electronics, Japan). A combination of axial T1-weighted, T2-weighted and fluid attenuated inversion recovery (FLAIR) images was used to evaluate CVD. Lesions were considered to be CVD when they demonstrated low intensity on the T1-weighted or FLAIR images and high intensity on the T2-weighted image at the same location. We operationally considered those changes with diameters $\geq 4$ $\mathrm{mm}$ as CVD (presence of cerebral infarction). The images were visually evaluated by two teams, which consisted of two neurologists and a psychiatrist, and a senior neurologist [12].

Diagnostic Criteria for Dementia and CDR 0.5 Subtypes

Dementia was diagnosed according to the Diagnostic and Statistical Manual of Mental Disorders, 4th Edition, (DSM-IV) criteria [25]. In this study, we divided CDR 0.5 subtypes into 2 groups: vmSVD and other types of dementia.

vmSVD. In accordance with Erkinjuntti et al. [26], participants who met the following criteria were diagnosed with SVD: a score on the memory item of the CDR of 0.5 or more, the presence of executive dysfunction (we used a mean performance time +1 SD on the TrailMaking Tests A or B stratified by age and educational levels [7]), 5 or more lacunar infarctions along with white matter lesions, the presence of neurologic signs, and the absence of cortical infarctions ( $8 \mathrm{~mm}$ and over) using MRI. The participants with SVD who scored a CDR of 0.5 were diagnosed with vmSVD in this study [11].

Other Types (of Dementia). We included all participants with a CDR score of 0.5 but no vmSVD in this study.

\section{Measures}

We used the Japanese version of the MoCA [15] (MoCA-J [17]) and MMSE [14] as neuropsychological tests for the assessment of all participants. In this study, the MoCA and MMSE were 30-point tests administered in 15 and 20 min, respectively. About MoCA scoring, if education was $\leq 12$ years, we added 1 point to the total score $[15,17]$. The MoCA consists of 8 subscales: visuospatial/executive function (alternating Trail-Making, cube copy, and clock-drawing task), naming (3 animals), memory (only repeat, no points), attention (forward and backward digit span, target detection using tapping, and a serial subtraction task), language (sentence repetition and verbal fluency), abstraction, delayed recall (5 nouns, after approximately $5 \mathrm{~min}$ ), and orientation (time and place) [15]. The cutoff point of the MoCA-J was $25 / 26$ (sensitivity $93 \%$ and specificity $87 \%$ ) in screening MCI ( $\mathrm{n}=30$, mean age 77.3 years, mean education 11.5 years) in a clinic-based study [17].

We used the Japanese version of MMSE [14] for assessing general cognitive functions, and compared MMSE with the MoCA. MMSE consists of orientation, registration, serial $7 \mathrm{~s}$, recall, naming, repetition, comprehension, reading, writing and drawing. The MMSE score significantly declined with age and lower educational level, although no effect of sex was apparent in healthy elderly in the Japanese community [27].

\section{Analyses}

Screening a CDR of 0.5 (MCI)

We compared the total MoCA score, the MoCA subscale scores and MMSE scores in 3 CDR groups. We used the $\chi^{2}$ test (significant level: $p<0.005$; d.f. $=2$ ) for sex, percentages of education $\leq 12$ years and presence of infarction, the one-way ANOVA $(\mathrm{p}<0.005$; d.f. $=2,389)$ with Bonferroni's post hoc test $(\mathrm{p}<0.05)$ for age, education and CDR-SOB. We used the oneway ANCOVA ( $p<0.005$; d.f. $=2$, 389; covariates: age and education, $p<0.05$ ) with Bonferroni's post hoc test $(\mathrm{p}<0.05)$ for the total MoCA, the MoCA subscales and MMSE scores. 
We calculated the area under the curve (AUC) of the receiver operating characteristic (ROC) curve (and its 95\% CI) of the MoCA and MMSE (CDR 0.5 vs. CDR 0; CDR 0.5 or more vs. CDR 0). Participants with a CDR of 0.5 or more included those with a CDR of 0.5 ( $\mathrm{n}=$ $184)$ and CDR of $1+(\mathrm{n}=44)$. Sensitivity, specificity, positive predictive values (PPV) and negative predictive values (NPV) as well as likelihood ratios were calculated with standard formulas. We compared the AUC of the MoCA with that of MMSE using the paired $z$ test [28].

Infarction Effect (Absence vs. Presence)

In this study, the presence of an infarction indicated cerebral infarctions $\geq 4 \mathrm{~mm}$ in size, and the absence of an infarction indicated $<4 \mathrm{~mm}$ or no cerebral infarctions on the MRI. Since almost half of our participants had one or more infarctions, we investigated the effect of cerebral infarction on the MoCA.

We divided CDR 0 participants into 2 groups: infarction present $(n=65)$ and absent $(\mathrm{n}=93)$, and CDR 0.5 participants into 2 groups: infarction present $(\mathrm{n}=96)$ and absent $(\mathrm{n}=$ 83). We compared the total MoCA, the MoCA subscales and MMSE scores using $2 \times 2 \mathrm{AN}$ COVA (CDR 0 vs. CDR $0.5 \times$ infarction present vs. infarction absent; $\mathrm{p}<0.005$; d.f. $=3,333$ ) with the covariates age and education $(\mathrm{p}<0.05)$.

Detecting vmSVD

We compared the total MoCA, the MoCA subscales and MMSE scores in the 3 groups: healthy elderly $(n=164)$ versus vmSVD $(n=37)$ versus other types $(n=147)$. We used the $\chi^{2}$ test (significant level, $\mathrm{p}<0.005$; d.f. $=2$ ) for sex and percentage of education $\leq 12$ years, and the one-way ANOVA $(\mathrm{p}<0.005$; d.f. $=2,345)$ with Bonferroni's post hoc test $(\mathrm{p}<0.05)$ for age, education and CDR-SOB. We used the one-way ANCOVA ( $<<0.005$; d.f. $=2$, 344; covariates: age and education, $p<0.05)$ with Bonferroni's post hoc test $(p<0.05)$ for the total MoCA, the MoCA subscales and MMSE scores.

We calculated the AUC of the ROC curve (and its 95\% CI) of the MoCA, MMSE and the combination of both the MoCA attention subscale and MMSE (vmSVD vs. CDR 0). We compared the AUC of the MoCA and the combination of both the MoCA attention subscale and MMSE with that of MMSE using the paired $z$ test [28].

\section{Ethics}

Written informed consent was obtained from every participant with a CDR of 0 and 0.5 and from the family of those with a CDR of 0.5 and those with dementia. The study was approved by the Ethics Committees of the Kurihara City government and Tohoku University Graduate School of Medicine.

\section{Results}

Screening a CDR of 0.5 (MCI)

Table 1 shows the demographic information and the mean scores of the total MoCA, the MoCA subscales and MMSE of the $3 \mathrm{CDR}$ groups. Among the demographic variables, there were significant differences between the 3 groups in age, education and CDR-SOB. The CDR 0.5 group had a significantly higher age and CDR-SOB score, and lower education than the CDR 0 group. Most of the participants had a low educational level $(\leq 12$ years, $382 / 392=$ $97.4 \%$; $<12$ years, $340 / 392=86.7 \%$ ).

In the neuropsychological tests, there were significant differences between the $3 \mathrm{CDR}$ groups in the total MoCA score, the MoCA subscale score including visuospatial and execu- 
in a Community: The Kurihara Project

Table 1. Demographics and the MoCA scores in the 3 CDR groups

\begin{tabular}{|c|c|c|c|c|c|c|c|}
\hline & \multirow{2}{*}{$\begin{array}{l}\text { CDR } 0 \\
\text { (healthy; } \\
\mathrm{n}=164 \text { ) }\end{array}$} & \multirow{2}{*}{$\begin{array}{l}\text { CDR } 0.5 \\
(\mathrm{MCI} ; \\
\mathrm{n}=183)\end{array}$} & \multirow{2}{*}{$\begin{array}{l}\text { CDR 1+ } \\
\text { (dementia; } \\
\mathrm{n}=44 \text { ) }\end{array}$} & \multirow{2}{*}{$\begin{array}{l}\mathrm{F} / \chi^{2} \\
\text { value }\end{array}$} & \multirow[t]{2}{*}{$\mathrm{p}$ value } & \multicolumn{2}{|c|}{ Covariance effects } \\
\hline & & & & & & $\mathrm{p}_{\text {value }}{ }^{1}$ & p value ${ }^{2}$ \\
\hline Females & $57.9 \%$ & $61.4 \%$ & $61.4 \%$ & 0.5 & 0.787 & - & - \\
\hline Age, years & $79.1(3.6)$ & $80.8(4.4)^{\mathrm{a}}$ & $82.5(4.2)^{\mathrm{a}, \mathrm{b}}$ & 14.4 & $<0.001$ & - & - \\
\hline Education, years & $9.4(2.0)$ & $8.5(1.7)^{\mathrm{a}}$ & $8.0(1.7)^{\mathrm{a}}$ & 15.6 & $<0.001$ & - & - \\
\hline Education $\leq 12$ years & $95.5 \%$ & $100 \%$ & $97.7 \%$ & 10.5 & 0.005 & - & - \\
\hline CDR-SOB & $0.3(0.4)$ & $1.5(0.9)^{\mathrm{a}}$ & $7.7(3.1)^{\mathrm{a}, \mathrm{b}}$ & 620.0 & $<0.001$ & - & - \\
\hline Presence of infarction & $41.1 \%$ & $53.6 \%$ & $59.5 \%$ & 7.3 & 0.026 & - & - \\
\hline MoCA total (full score: $30^{\#}$ ) & $21.1(4.0)$ & $17.5(4.5)^{\mathrm{a}}$ & $11.5(5.6)^{\mathrm{a}, \mathrm{b}}$ & 62.1 & $<0.001$ & 0.044 & $<0.001$ \\
\hline \multicolumn{8}{|l|}{ MoCA subscales (full score) } \\
\hline Visuospatial/executive (5) & $3.4(1.2)$ & $2.8(1.2)^{\mathrm{a}}$ & $1.9(1.5)^{\mathrm{a}, \mathrm{b}}$ & 16.9 & $<0.001$ & 0.337 & $<0.001$ \\
\hline Naming (3) & $2.7(0.5)$ & $2.4(0.8)^{\mathrm{a}}$ & $1.8(0.9)^{\mathrm{a}, \mathrm{b}}$ & 15.4 & $<0.001$ & 0.289 & 0.002 \\
\hline Attention (6) & $4.3(1.4)$ & $3.2(1.8)^{\mathrm{a}}$ & $2.1(1.7)^{\mathrm{a}, \mathrm{b}}$ & 23.3 & $<0.001$ & 0.262 & $<0.001$ \\
\hline Language (3) & $0.7(0.8)$ & $0.5(0.7)$ & $0.3(0.6)$ & 1.8 & 0.174 & 0.769 & $<0.001$ \\
\hline Abstraction (2) & $1.4(0.8)$ & $1.0(0.8)^{\mathrm{a}}$ & $0.6(0.8)^{\mathrm{a}, \mathrm{b}}$ & 11.9 & $<0.001$ & 0.502 & $<0.001$ \\
\hline Delayed recall (5) & $2.0(1.8)$ & $0.9(1.4)^{\mathrm{a}}$ & $0.3(0.8)^{\mathrm{a}}$ & 22.3 & $<0.001$ & 0.022 & 0.133 \\
\hline Orientation (6) & $5.9(0.4)$ & $5.6(0.7)$ & $3.6(1.9)^{\mathrm{a}, \mathrm{b}}$ & 106.5 & $<0.001$ & 0.049 & 0.163 \\
\hline MMSE (full score: 30 ) & $25.2(2.6)$ & $22.9(3.6)^{\mathrm{a}}$ & $16.0(5.7)^{\mathrm{a}, \mathrm{b}}$ & 93.6 & $<0.001$ & 0.255 & $<0.001$ \\
\hline \multicolumn{8}{|c|}{$\begin{array}{l}\text { Statistical analyses: sex, education } \leq 12 \text { years and presence of infarction: } \chi^{2} \text { test }(\mathrm{p}<0.005) \text {; age, education, and CDR-SOB: } \\
\text { e-way ANOVA }(\mathrm{p}<0.005) \text {, Bonferroni's post hoc test }(\mathrm{p}<0.05) \text {; MoCA and MMSE: one-way ANCOVA }(\mathrm{p}<0.005 \text {; covari- } \\
\text { es: age and education, } \mathrm{p}<0.05) \text {, Bonferroni's post hoc test }(\mathrm{p}<0.05) \text {. } \\
\text { a Significantly lower (age, SOB; higher) than the CDR } 0 \text { group }(\mathrm{p}<0.05) \text {. } \\
{ }^{\mathrm{b}} \text { Significantly lower (age, SOB; higher) than the CDR } 0.5 \text { group }(\mathrm{p}<0.05) \text {. }\end{array}$} \\
\hline
\end{tabular}

tive function, naming, attention, abstraction, delayed recall and orientation, and MMSE score. In all of them, the CDR 0 group had higher scores than the CDR 0.5 group, which had higher scores than the CDR 1+ group except for delayed recall.

Cutoff Score of CDR 0.5 or More versus CDR 0

The AUC of the ROC curves of the MoCA and MMSE were 0.76 ( $\mathrm{p}<0.001,95 \%$ CI $0.72-0.81)$ and $0.74(\mathrm{p}<0.001,95 \%$ CI $0.69-0.79)$, respectively. The cutoff scores of the MoCA and MMSE were $19 / 20$ and $24 / 25$, sensitivity was 71.5 and $73.2 \%$, and specificity 66.5 and $59.0 \%$, respectively; the PPV were 74.8 and $71.1 \%$, and the NPV 62.6 and $61.1 \%$, respectively. The positive likelihood ratios were 2.13 and 1.79 , and the negative likelihood ratios 0.43 and 0.45 , respectively (fig. $2 \mathrm{a}$ ). There was no significant difference between the AUC of the MoCA and MMSE $(\mathrm{Z}=1.09, \mathrm{p}=0.276)$ using $\mathrm{Z}$-score analysis.

Cutoff Score of CDR 0.5 versus CDR 0

The AUC of the ROC curves of the MoCA and MMSE were $0.73(\mathrm{p}<0.001,95 \%$ CI $0.68-0.78)$ and $0.70(\mathrm{p}<0.001,95 \%$ CI $0.64-0.75)$, respectively. The cutoff scores of the MoCA and MMSE were $19 / 20$ and 24/25, sensitivity was 66.3 and $68.5 \%$, and specificity 66.5 and $58.5 \%$, respectively; the PPV were 68.9 and $64.9 \%$, and the NPV 63.7 and $62.3 \%$, respectively. The positive likelihood ratios were 1.98 and 1.65 , and the negative likelihood ratios 
Kasai et al.: Screening for vmSVD Patients Aged 75 and Above Using the MoCA and MMSE in a Community: The Kurihara Project

Fig. 2. ROC analysis. a MoCA versus MMSE. ROC of the MoCA (thin black line) and MMSE (dashed black line) to distinguish participants with a CDR of 0.5 and more from those with a CDR of 0 . $\mathbf{b}$ MoCA versus MMSE versus the combination of both MoCA attention subscale and MMSE. ROC of the MoCA (thin black line), MMSE (dashed black line), and the combination of both the MoCA attention subscale and MMSE (thick black line) to distinguish participants with vmSVD from those with a CDR of 0 .
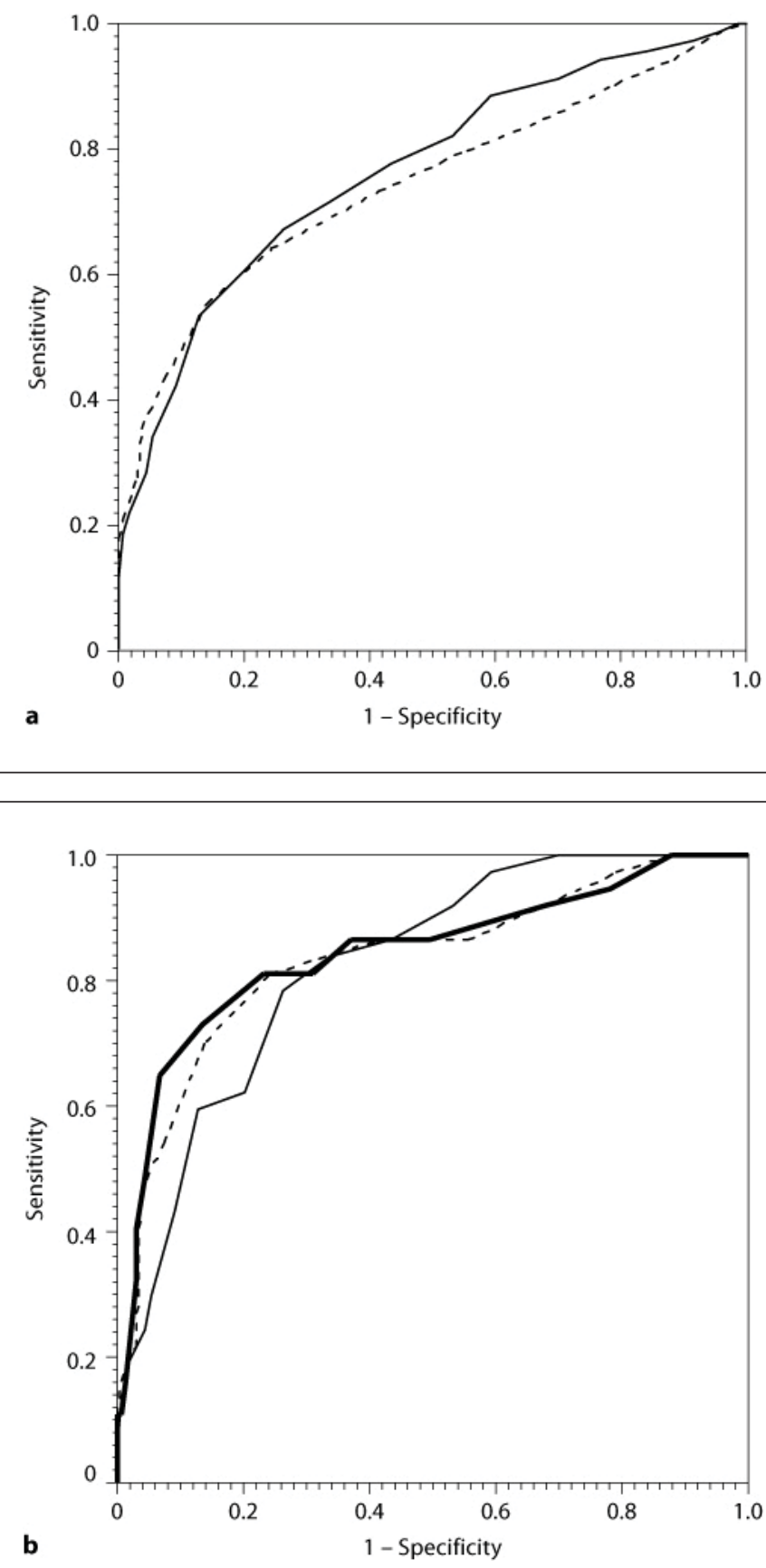

0.51 and 0.54 , respectively. However, on the histogram, the MoCA scores overlapped with CDR 0 and CDR 0.5 participants (fig. 3). There was no significant difference between the AUC of the MoCA and MMSE $(\mathrm{Z}=1.37, \mathrm{p}=0.170)$.

Infarction Effect (Absence vs. Presence)

Looking at the demographics, there was no significant difference in the percentage of females between CDR 0 and CDR 0.5 participants (CDR 0 with infarction $50.8 \%$ vs. without 
Fig. 3. Histogram of the participants with a CDR of 0 and a CDR of 0.5 .

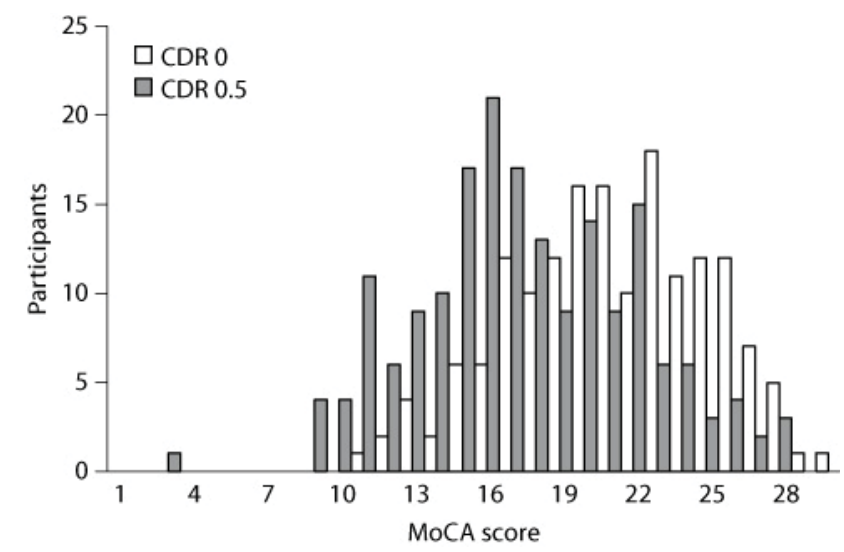

64.5\%; CDR 0.5 with infarction $57.3 \%$ vs. without $65.1 \%)$. For $2 \times 2$ ANOVA, there were significant CDR effects on age $(\mathrm{F}=12.0, \mathrm{p}=0.001)$, education $(\mathrm{F}=12.0, \mathrm{p}<0.001)$ and CDRSOB $(\mathrm{F}=243.9, \mathrm{p}<0.001)$; however, there were no significant infarction effects on any of them $(\mathrm{F}=5.6,0.7$ and $0.1, \mathrm{p}=0.018,0.415$ and 0.723 , respectively).

In the neuropsychological tests, there were significant CDR effects on the total MoCA $(\mathrm{F}=36.1, \mathrm{p}<0.001 ;$ age effect, $\mathrm{p}=0.025$; education effect, $\mathrm{p}<0.001)$ and MMSE $(\mathrm{F}=24.5$, $\mathrm{p}<0.001$; education effect, $\mathrm{p}<0.001)$; however, there were no significant infarction effects on any of the scores ( $F=0.8$ and $0.1, p=0.379$ and 0.921 , respectively). In the MoCA subscale, there were significant $\mathrm{CDR}$ effects on visuospatial/executive function $(\mathrm{F}=8.6, \mathrm{p}=0.004$; education effect, $\mathrm{p}<0.001)$, attention $(\mathrm{F}=17.1, \mathrm{p}<0.001$; education effect, $\mathrm{p}<0.001)$ and delayed recall $(\mathrm{F}=28.1, \mathrm{p}<0.001$; age effect, $\mathrm{p}=0.028)$; however, there were no significant infarction effects on any of the subscale scores $(\mathrm{F}=1.6,1.8$ and $0.5, \mathrm{p}=0.212,0.180$ and 0.494 , respectively).

\section{Detecting vmSVD}

Looking at the demographics, there was no significant difference in the percentage of females among the healthy, the vmSVD and the other types groups ( 57.9 vs. 54.1 vs. $63.3 \%$, $\mathrm{F}=1.5, \mathrm{p}=0.477)$. There were significant differences between the 3 groups in age $(\mathrm{F}=9.0$, $\mathrm{p}<0.001)$, education $(\mathrm{F}=10.0, \mathrm{p}<0.001)$ and CDR-SOB $(\mathrm{F}=138.9, \mathrm{p}<0.001)$. The vmSVD group had a significantly higher age and SOB score, and lower education level than the healthy elderly group but not the other types group.

In the neuropsychological tests, there were significant differences between the 3 groups in the total MoCA score (mean: 21.1 vs. 16.0 vs. $17.8 ; \mathrm{F}=22.4, \mathrm{p}<0.001$; education effect, $\mathrm{p}<0.001$ ), the MoCA subscale score including visuospatial/executive function (mean: $3.4 \mathrm{vs}$. 2.6 vs. $2.8 ; \mathrm{F}=6.0, \mathrm{p}=0.003$; education effect, $\mathrm{p}<0.001$ ), attention (mean: 4.3 vs. 2.5 vs. 3.4; $\mathrm{F}=15.5, \mathrm{p}<0.001$; education effect, $\mathrm{p}<0.001$ ), delayed recall (mean: 2.0 vs. 0.5 vs. $1.0 ; \mathrm{F}=$ 15.2 , p < 0.001) and MMSE score (mean: 25.2 vs. 21.4 vs. 23.3; $\mathrm{F}=19.4, \mathrm{p}<0.001$; education effect, $\mathrm{p}<0.001)$. The vmSVD group had lower scores than the other types group, which had lower scores than the healthy group in the total MoCA, the MoCA attention subscale and MMSE. The vmSVD and the other types groups had lower scores than the healthy elderly group in visuospatial/executive function and delayed recall. Figure 4 shows the MoCA total score (fig. $4 \mathrm{a}$ ) and the MoCA attention subscale score (fig. 4b) of the 3 groups. 
Fig. 4. The total MoCA and the MoCA attention subscale scores in healthy elderly, vmSVD and other types participants. a The total MoCA scores in the 3 groups. b The MoCA attention subscale scores in the 3 groups. ${ }^{*} \mathrm{p}=0.05$.

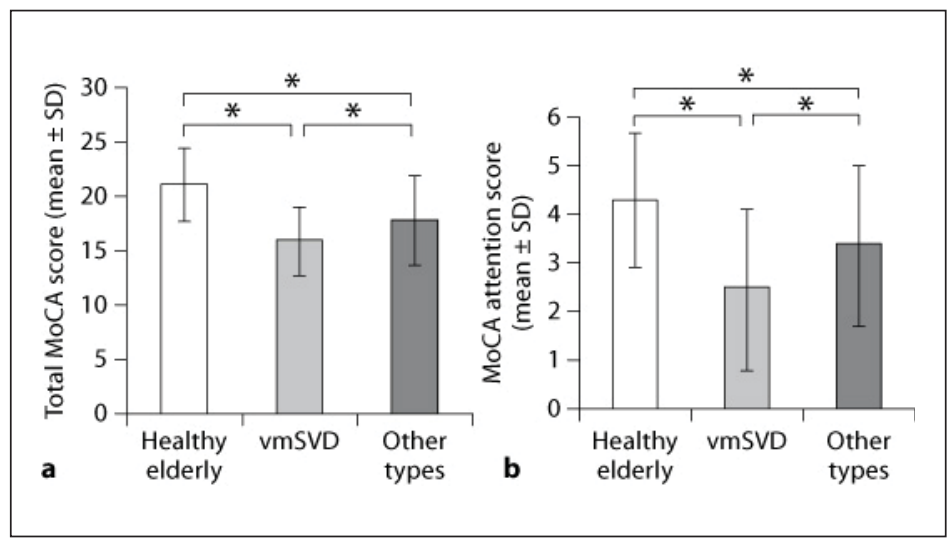

Cutoff Score of vmSVD versus CDR 0

The AUC of the ROC curves of the MoCA, MMSE and the combination of both the MoCA attention subscale and MMSE (full score 36) were 0.83 ( $p<0.001,95 \%$ CI $0.76-0.89$ ), 0.84 ( $\mathrm{p}<0.001,95 \%$ CI $0.76-0.91)$ and 0.84 ( $\mathrm{p}<0.001,95 \%$ CI $0.76-0.93)$, respectively. The cutoff scores of them were $18 / 19,23 / 24$ and $26 / 27$, sensitivity was $78.4,81.1$ and $81.1 \%$, and specificity $73.8,75.6$ and $76.8 \%$, respectively; the PPV were $40.3,42.9$ and $44.1 \%$, and the NPV 93.8, 94.7 and 94.7\%, respectively. The positive likelihood ratios were 2.99, 3.32 and 3.50 , and the negative likelihood ratios $0.29,0.25$ and 0.25 , respectively (fig. $2 \mathrm{~b}$ ). There were no significant differences between the AUC of the MoCA and MMSE $(Z=-0.28, p=0.779)$, and those of the combination of both the MoCA attention subscale and MMSE ( $Z=0.55$, $\mathrm{p}=0.960)$.

\section{Discussion}

In this study, there was a significant difference in the MoCA and MMSE scores between the 3 CDR groups (CDR $0>$ CDR $0.5>$ CDR $1+$ ). However, the MoCA score overlapped in CDR 0 and CDR 0.5 participants. There were significant CDR effects, while there were no significant infarction effects for the MoCA and MMSE $(2 \times 2$ ANCOVA). vmSVD participants had significantly lower scores of the total MoCA, the MoCA attention subscale and MMSE than healthy elderly and other types participants. The total MoCA and the MoCA attention subscale may detect the characteristics of vmSVD in the elderly.

Screening a CDR of 0.5 (MCI)

There was a significant difference in the total MoCA and MMSE scores between the 3 CDR groups (CDR $0>C D R 0.5>C D R 1+$ ). Our findings are consistent with previous studies using MCI criteria in a community [16]. In our results, the AUC of the ROC curves (CDR 0.5 and more vs. CDR 0) of the MoCA was slightly larger than that of MMSE; however, the MoCA scores overlapped in the CDR 0 and CDR 0.5 participants on the histogram. Our cutoff point was less than 20, and it was lower than in previous studies [15-17]. Previous studies showed several cutoff points in healthy elderly and MCI participants; cutoff score 1 was $\leq 23$ in the community-based study [16], and cutoff score 2 was $<26$ in the clinic-based studies in Canada [15] and Japan [17]. The reason why our MoCA score was lower than in previous studies was that our participants had old-old age ( $75+$ years) and low education ( $\leq 12$ years: $97.4 \%$; $<12$ years: $86.7 \%$ ). In previously published normative data, the MoCA score in the age 
Table 2. MoCA (raw score) by educational level in healthy elderly

\begin{tabular}{|c|c|c|c|c|c|c|c|c|c|c|}
\hline \multirow[t]{2}{*}{ Age group } & \multicolumn{2}{|c|}{$\begin{array}{l}\leq 6 \text { years of } \\
\text { education }\end{array}$} & \multicolumn{2}{|c|}{$\begin{array}{l}7-9 \text { years of } \\
\text { education }\end{array}$} & \multicolumn{2}{|c|}{$\begin{array}{l}10-12 \text { years of } \\
\text { education }\end{array}$} & \multicolumn{2}{|c|}{$\begin{array}{l}\geq 13 \text { years of } \\
\text { education }\end{array}$} & \multicolumn{2}{|c|}{ Total } \\
\hline & $\mathrm{n}$ & mean $\pm \mathrm{SD}$ & $\mathrm{n}$ & mean $\pm \mathrm{SD}$ & $\mathrm{n}$ & mean $\pm \mathrm{SD}$ & $\mathrm{n}$ & mean $\pm \mathrm{SD}$ & $\mathrm{n}$ & mean $\pm S D$ \\
\hline 75-90 years & 8 & $16.4 \pm 3.2$ & 106 & $19.5 \pm 3.5$ & 41 & $22.0 \pm 4.4$ & 9 & $23.6 \pm 4.7$ & 164 & $20.2 \pm 4.1$ \\
\hline
\end{tabular}

$\leq 6$ years = Elementary school; $7-9$ years $=$ junior high school; $10-12$ years = high school; $\geq 13$ years = university (in the Japanese old education system).

ANOVA: $\mathrm{F}=9.26, \mathrm{p}<0.001$; Bonferroni's post hoc test $(\mathrm{p}<0.05): \leq 6$ years $=7-9$ years $<10-12$ years $=\geq 13$ years.

group 70-80 years with $<12$ years of education was $16.07(\mathrm{n}=14)$, and with 12 years of education it was 20.35 ( $n=23$; no 1-point correction, respectively) [18]. Table 2 shows the MoCA (raw score) by educational level in healthy elderly. In participants educated for $\geq 13$ years, our data and the previous study's data [18] yielded almost the same MoCA score. Meanwhile, in people educated for 10-12 and 7-9 years, our data resulted in a higher MoCA score than the previous study's data ( 22.0 vs. 20.35 and 19.5 vs. 16.1) [18]. One of the reasons may be that groups with a low educational level may be culturally different. Since our population consisted of a relatively large number of participants aged $75+$ and had low education, it contributes to the normative data in a community-based study.

\section{Infarction Effect (Absence vs. Presence)}

Nearly half of our participants had cerebral infarction due to the fact that they were oldold people living in a rural community. In our results, there were significant CDR effects but no significant infarction effects on the MoCA and MMSE. However, a few participants with large cortical infarctions ( $\mathrm{n}=12,>8 \mathrm{~mm}$ on MRI) had lower MoCA scores than participants without infarctions. As a reference for discussion, the mean (SD) MoCA total score of CDR 0 participants with large cortical infarctions $(n=4)$ was $17.0(3.7)$, and that of CDR 0.5 participants with large cortical infarctions $(n=8)$ was $14.3(3.5)$. We found lesions of large cortical infarctions at the following locations: right and left anterior cerebral artery hemispheric brain and pericallosal brain, right and left middle cerebral artery (MCA) hemispheric brain, right MCA penetrating brain and left posterior inferior cerebellar artery. Since there was a very small number of participants with large cortical infarctions in this study, this will need to be investigated in the future.

\section{Detecting vmSVD}

vmSVD participants had significantly lower total MoCA, MoCA attention subscale and MMSE scores than healthy elderly and other types of dementia with CDR 0.5. This result suggests that the MoCA and MMSE, especially the MoCA attention subscale, are effective in detecting characteristics of people with VmSVD opposed to healthy elderly and other types of dementia with CDR 0.5. The total MoCA, MMSE and the combination of both the MoCA attention subscale and MMSE are good screening tests for detecting vmSVD; however, there were no significant differences in the AUC of the ROC curves among these tests in our study.

The MoCA attention subscale contains forward and backward digit span tests. Swartz et al. [29] used forward and backward digit span tests as part of their working memory and attention tasks, for brain measurements of normal, cognitive impairment no dementia and vas- 
cular dementia participants. They reported that large cortical strokes and anterior-medical thalamic hypersensitivity were related to working memory/attention and short-term memory, i.e. the thalamico-cortical network [29]. It is therefore likely that the MoCA is able to detect participants with deficits in attention and working memory, such as participants with vmSVD.

\section{Limitations}

We used Erkinjuntti et al.'s [26] criteria for vmSVD participants. Although based on Erkinjuntti et al.'s criteria, the term 'very mild subcortical vascular dementia (vmSVD)' is debatable as it includes both vascular MCI and vascular dementia; both are now grouped under the term 'vascular cognitive impairment (VCI)' [30]. However, since vmSVD patients have some vascular risk factors, they could be therapeutically objective. The measurement of executive dysfunction in Erkinjuntti et al. is not clear. In this study, executive dysfunction was examined using Trail-Making Tests A and B, and this may be a limitation of the study. However, these tests have been validated in part as indexes of the assessment of executive functions [31]. The MoCA visuospatial/executive subscale contains a short version of the Trail-Making Test B. However, in our study, there was no significant association in the MoCA visuospatial/executive subscale between participants with vmSVD and other types of dementia.

The grouping according to 'presence of infarction' was an operational definition using MRI in this study. In the future, the association between the MoCA scores and focal brain lesions needs to be assessed in all participants.

It is worthy to consider categorizing the MoCA subscales. It would be prudent to point out that these are derived via clinical intuition, and in fact the argument could be made that some items could be considered to fall into more than one subscale. For example, some would consider verbal fluency to be also an executive function task [31], and naming to be also a language task.

\section{Conclusions}

We recommend to use the MoCA carefully as a reference test to detect CDR 0.5 people who are aged $75+$ and have low education $(<12$ years) in a community. Our results suggest that MMSE performed rather well and that the MoCA is not superior to MMSE in MCI and vmSVD participants aged $75+$ in a community. We will perform further research in a clinicbased study on participants with CVD and low MoCA scores, and in a longitudinal study on participants with dementia predictors.

\section{Acknowledgement}

We are grateful to the staff at Kurihara City government, especially to the public health nurses, and the staff at the Department of Geriatric Behavioral Neurology, Tohoku University Graduate School of Medicine. In particular, we thank Dr. Kyoko Akanuma, Mses Yuriko Kato, Megumi Nakai, Madoka Hasebe and Yoko Saito.

\section{Disclosure Statement}

The authors have no conflicts of interest to disclose. 


\section{References}

al et al: Screening for vmSVD Patients Aged 75 and Above Using the MoCA and MMSE in a Community: The Kurihara Project

1 Flicker C, Ferris SH, Reisberg B: Mild cognitive impairment in the elderly: predictors of dementia. Neurology 1991;41:1006-1009.

-2 Petersen RC, Smith GE, Waring SC, Ivnik RJ, Tangalos EG, Kokmen E: Mild cognitive impairment: clinical characterization and outcome. Arch Neurol 1999;56:303-308.

-3 Hughes CP, Berg L, Danziger WL, Coben LA, Martin RL: A new clinical scale for the staging of dementia. Br J Psychiatry 1982;140:566-572.

4 Morris JC: The Clinical Dementia Rating (CDR): current version and scoring rules. Neurology 1993; 43:2412-2414.

-5 Hashimoto R, Meguro K, Yamaguchi S, Ishizaki J, Ishii H, Meguro M, Sekita Y: Executive dysfunction can explain word-list learning disability in very mild Alzheimer's disease: the Tajiri project. Psychiatry Clin Neurosci 2004;58:54-60.

-6 Kasai M, Meguro K, Hashimoto R, Ishizaki J, Yamadori A, Mori E: Non-verbal learning is impaired in very mild Alzheimer's disease (CDR 0.5): normative data from the learning version of the ReyOsterrieth Complex Figure Test. Psychiatry Clin Neurosci 2006;60:139-146.

-7 Hashimoto R, Meguro K, Yamaguchi S, Ishizaki J, Ishii H, Meguro M, Sekita Y: Executive dysfunction can explain word-list learning disability in very mild Alzheimer's disease: the Tajiri project. Psychiatry Clin Neurosci 2004;58:54-60.

8 Kasai M, Ishizaki J, Meguro K: Alzheimer's patients do not show left unilateral spatial neglect but exhibit peripheral inattention and simplification. Dement Neuropsychol 2007;1:374-380.

-9 Kasai M, Ishizaki J, Ishii H, Yamaguchi S, Yamadori A, Meguro K: Normative data on Benton Visual Form Discrimination Test for older adults and impaired scores in Clinical Dementia Rating 0.5 participants: community-based study. The Osaki-Tajiri Project. Psychiatry Clin Neurosci 2009;63: 9-16.

-10 Ingles JL, Wentzel C, Fisk JD, Rockwood K: Neuropsychological predictors of incident dementia in patients with vascular cognitive impairment, without dementia. Stroke 2002;33:1999-2002.

-11 Meguro K, Akanuma K, Meguro M, Kasai M, Ishii H, Yamaguchi S: Prognosis of vascular mild cognitive impairment includes vascular dementia onset and death by cardiovascular disease: reanalysis from the Osaki-Tajiri Project. J Stroke Cerebrovasc Dis 2012;21:607-611.

-12 Ishii H, Meguro K, Yamaguchi S, Ishikawa H, Yamadori A: Prevalence and cognitive performances of vascular cognitive impairment no dementia in Japan: the Osaki-Tajiri Project. Eur J Neurol 2007; 14:609-616.

-13 Nakata E, Kasai M, Kasuya M, Akanuma K, Meguro M, Ishii H, Yamaguchi S, Meguro K: Combined memory and executive function tests can screen mild cognitive impairment and converters to dementia in a community: the Osaki-Tajiri project. Neuroepidemiology 2009;33:103-110.

-14 Folstein MF, Folstein SE, McHugh PR: 'Mini-mental state': a practical method for grading the cognitive state of patients for the clinician. J Psychiatr Res 1975;12:189-198.

- 15 Nasreddine ZS, Philips NA, Bedirian V, Charbonneau S, Whitehead V, Collin I, Cummins JL, Chertkow H: The Montreal Cognitive Assessment, MoCA: a brief screening tool for mild cognitive impairment. J Am Geriatr Soc 2005;53:695-699.

-16 Luis CA, Keegan AP, Mullan M: Cross-validation of the Montreal Cognitive Assessment in community dwelling older adults residing in the southeastern USA. Int J Geriatr Psychiatry 2009;24:197-201.

-17 Fujiwara Y, Suzuki H, Yasunaga M, Sugiyama M, Ijuin M, Sakuma N, Inagaki H, Iwasa H, Ura C, Yatomi N, Ishii K, Tokumaru AM, Homma A, Nasreddine Z, Shinkai S: Brief screening tool for mild cognitive impairment in older Japanese: validation of the Japanese version of the Montreal Cognitive Assessment. Geriatr Gerontol Int 2010;10:225-232.

-18 Rossetti HC, Lacritz LH, Cullum CM, Weiner MF: Normative data for the Montreal Cognitive Assessment (MoCA) in a population-based sample. Neurology 2011;77:1272-1275.

-19 Nasreddine ZS, Phillips N, Chertkow H, Rossetti H, Lacritz L, Cullum M, Weiner M: Normative data for the Montreal Cognitive Assessment (MoCA) in a population-based sample. Neurology 2012;78: $765-766$.

-20 Sweet L, Van Adel M, Metcalf V, Wright L, Harley A, Leiva R, Taler V: The Montreal Cognitive Assessment (MoCA) in geriatric rehabilitation: psychometric properties and association with rehabilitation outcomes. Int Psychogeriatr 2011;23:1582-1591. 
21 Lezak MD, Howieson DB, Loring DW: Neuropsychological Assessment, ed 4. New York, Oxford University, 2004.

-22 Godefroy O, Fickl A, Roussel M, Auribault C, Bugnicourt JM, Lamy C, Canaple S, Petitnicolas G: Is the Montreal Cognitive Assessment superior to the Mini-Mental State Examination to detect poststroke cognitive impairment? A study with neuropsychological evaluation. Stroke 2011;42:17121716.

23 Ihara M, Okamoto Y, Takahashi R: Suitability of the Montreal Cognitive Assessment versus the Mini-Mental State Examination in detecting vascular cognitive impairment. J Stroke Cerebrovasc Dis 2012 [Epub ahead of print].

-24 Meguro K, Ishii H, Yamaguchi S, Ishizaki J, Shimada M, Sato M, Hashimoto R, Shimada Y, Meguro M, Yamadori A, Sekita Y: Prevalence of dementia and dementing diseases in Japan: the Tajiri project. Arch Neurol 2002;59:1109-1114.

25 American Psychiatric Association: Diagnostic and Statistical Manual of Mental Disorders (DSMIV), ed 4. Washington, American Psychiatric Association, 1994.

- 26 Erkinjuntti T, Inzitari D, Pantoni L, Wallin A, Scheltens P, Rockwood K, Roman GC, Chui H, Desmond DW: Research criteria for subcortical vascular dementia in clinical trials. J Neural Transm Suppl 2000;59:23-30.

-27 Ishizaki J, Meguro K, Ambo H, Shimada M, Yamaguchi S, Hayasaka C, Komatsu H, Sekita Y, Yamadori A: A normative, community-based study of Mini-Mental State in elderly adults: the effect of age and educational level. J Gerontol B Psychol Sci Soc Sci 1998;53:359-363.

- 28 Hanley JA, McNeil BJ: A method of comparing the areas under receiver operating characteristic curves derived from the same cases. Radiology 1983;148:839-843.

-29 Swartz RH, Stuss DT, Gao F, Black SE: Independent cognitive effects of atrophy and diffuse subcortical and thalamico-cortical cerebrovascular disease in dementia. Stroke 2008;39:822-830.

- 30 Hachinski V, Iadecola C, Petersen RC, Breteler MM, Nyenhuis DL, Black SE, Powers WJ, DeCarli C, Merino JG, Kalaria RN, Vinters HV, Holtzman DM, Rosenberg GA, Wallin A, Dichgans M, Marler JR, Leblanc GG: National Institute of Neurological Disorders and Stroke-Canadian Stroke Network vascular cognitive impairment harmonization standards. Stroke 2006;37:2220-2241.

- 31 Godefroy O, Azouvi P, Robert P, Roussel M, LeGall D, Meulemans T; Groupe de Réflexion sur l'Evaluation des Fonctions Exécutives Study Group: Dysexecutive syndrome: diagnostic criteria and validation study. Ann Neurol 2010;68:855-864. 